\title{
Estudo da ecologia trófica do quelônio Podocnemis unifilis da região do baixo Xingu, utilizando análise isotópica de C e N
}

A análise isotópica tem se tornado uma ferramenta muito útil para os ecólogos e pesquisadores que estudam o ciclo da matéria. Os isótopos de $\mathrm{C}$ e $\mathrm{N}$ foram utilizados para demonstrar características ecológicas da espécie Podocnemis unifilis na região do Baixo Xingu, apresentando a análise isotópica como um método complementar em estudos ecológicos. Foram coletadas amostras de tecidos de 63 indivíduos de P. unifilis, sendo 34 machos e 29 fêmeas em quatro áreas. Através da análise isotópica verificamos possíveis variações intraespecíficas (relacionadas ao sexo e massa corporal) nos níveis tróficos e fontes primárias de carbono de suas dietas. Não foram apresentadas diferenças significativas ou padrões de absorção de $\mathrm{C}$ e $\mathrm{N}$ em relação ao tamanho do corpo e sexo. Porém foram encontradas diferenças no valor isotópico entre áreas com diferentes fisionomias.

Palavras-chave: Podocnemis unifilis; Isótopos estáveis; Dieta; Nível Trófico.

\section{Study of the trophic ecology of the chelonian Podocnemis unifilis from the lower Xingu region, using isotopic analysis of $\mathrm{C}$ and $\mathrm{N}$}

Isotopic analysis has become a very useful tool for ecologists and researchers studying the cycle of matter. Isotopic fractionation can bring an understanding of
how matter passes from one trophic level to another in an ecosystem. The $\mathrm{C}$ and $\mathrm{N}$ isotopes were used to demonstrate ecological characteristics of the species
Podocnemis unifilis in the Lower Xingu region, presenting the isotopic analysis as a complementary method in ecological studies. Tissue samples were collected
from 63 individuals of $\mathrm{P}$. unifilis, with 34 males and 29 females in four areas. Through the isotopic analysis, we verify possible intraspecific variations (related to sex
and body mass) in the trophic levels and primary sources of carbon of their diets. No significant differences or absorption patterns of $\mathrm{C}$ and $\mathrm{N}$ were presented in
relation to body size and sex. However, differences in isotopic value were found between areas with different physiognomies.

Keywords: Podocnemis unifilis; Stable isotopes; Diet; Trophic level.

Topic: Conservação da Biodiversidade

Reviewed anonymously in the process of blind peer.
Received: 04/02/2020

Approved: 05/03/2020
Douglas de Souza Xavier (D)

Universidade Federal Fluminense, Brasil http://lattes.cnpq.br/4291540940684628 http://orcid.org/0000-0002-9672-6687

douglasreivax@hotmail.com

José Antônio Baptista Neto (1)

Universidade Federal Fluminense, Brasil http://lattes.cnpq.br/6724474842271778 http://orcid.org/0000-0002-3638-4435 jabneto@id.uff.br

Adriana Malvasio

Universidade Federal do Tocantins, Brasil http://lattes.cnpq.br/9694032726460437 http://orcid.org/0000-0001-8020-3307 malvasio@mail.uft.edu.br

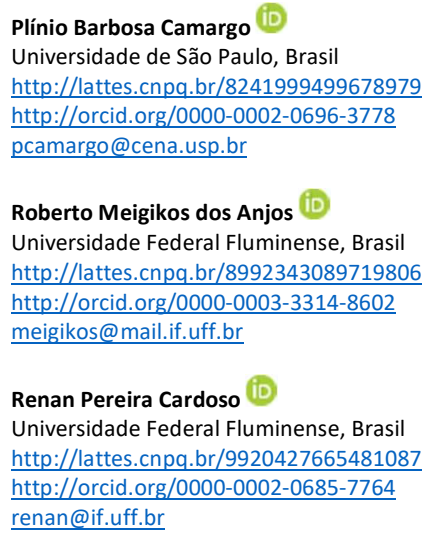

João Paulo de Sá Felizardo (iD)

Universidade Federal Fluminense, Brasil http://lattes.cnpq.br/3169099176102277 http://orcid.org/0000-0001-6579-7071 joaopaulo.sa.felizardo@gmail.com
Referencing this:

XAVIER, D. S.; BAPTISTA NETO, J. A.; MALVASIO, A.; CAMARGO, P. B.; ANJOS, R. M.; CARDOSO, R. P.; FELIZARDO, J. P. S.. Estudo da ecologia trófica do quelônio Podocnemis unifilis da região do baixo Xingu, utilizando análise isotópica de $\mathrm{C}$ e N. Revista Ibero Americana de Ciências Ambientais, v.11, n.2, p.99-109, 2020. DOI: http://doi.org/10.6008/CBPC2179-6858.2020.002.0012 


\section{INTRODUÇÃO}

O tracajá (Podocnemis unifilis Troschel) é uma das espécies de quelônios amazônicos mais abundantes, possuindo um tamanho médio corporal entre as espécies da família Podocnemididae e uma distribuição ampla nos rios da região norte do Brasil, centro-oeste e em grande parte dos rios dos países da América do Sul como, Bolívia, Peru, Guiana Francesa e Venezuela (FACHÍN-TERÁN et al., 2004; FACHÍN-TERÁN et al., 2003).

A conservação dos quelônios amazônicos é importante para manter a biodiversidade do bioma amazônico (BALESTRA et al., 2016) assim como o contexto histórico e atual, relacionados ao seu nicho ecológico e o seu papel como fonte de alimentação de algumas populações indígenas e ribeirinhas (FACHíNTERÁN et al., 2004; VOGT, 1994; REBÊLO et al., 1996). Na bacia amazônica as áreas inundáveis e/ou alagáveis apresentam uma grande biodiversidade, que é influenciada diretamente pelo padrão sazonal, as variações do nível da água podem atingir a 15 metros, modificando o habitat e influenciando todo o ecossistema amazônico, esse evento natural é denominado pulso de inundação (JUNK et al., 2004).

Existem registros da exploração desse grupo desde os tempos coloniais, através do consumo da carne, ovos e também gordura utilizadas como combustível para iluminação pública (BATES, 1892; MATOS, 1895; PORTELINHA, 2010). Atualmente as populações de quelônios amazônicos têm sofrido com as alterações de seus habitats reprodutivos e alimentares, assim com o aumento da pressão de caça (REBÊLO et al., 2000). Ainda hoje esse consumo desordenado dos recursos ociosos dos quelônios tem sido grande motivo de preocupação (VANZOLINE, 2003).

O P. unifilis atualmente consta com status vulnerável na IUCN Red List of Threatened Species, por esse motivo necessita de uma urgente atenção em estudo de conservação, pois é uma espécie de alta importância cultural, econômica e de equilíbrio ecológico dos ecossistemas amazônicos (VAN DIJK et al., 2014; PORTELINHA, 2010). Os quelônios são uma importante fonte de alimentação para as comunidades amazônicas (PANTOJA-LIMA et al., 2014; RÊBELO et al., 2000).

O estudo de ecologia, usando análise isotópica como ferramenta para determinar a posição trófica e a origem de recursos de nutrientes para populações da fauna silvestre, vem a cada dia sendo mais comum, investigando isótopos de carbono e nitrogênio incorporados nos tecidos animais (DENIRO et al., 1981; HOBSON et al., 1992). Até o momento para a classe dos répteis o grupo mais estudado são as tartarugas marinhas (HATASE et al., 2002; BIASATTI, 2004; WALLACE et al., 2006, SAMPSON et al., 2017), contudo vem crescendo o interesse em estudos com tartarugas de água doce (SEMINOFF, 2007; LARA et al., 2012). Lemons et al. (2011), através do estudo isotópico, identifica posição trófica das tartarugas verdes na Baía de San Diego, reforçando o conhecimento da ecologia dessa espécie em extinção, atualizando dados de forrageamento e nicho ecológico.

Alguns trabalhos fornecem informações de que os tecidos dos organismos refletem as composições isotópicas tanto de carbono quanto a nitrogênio em seus habitats (TOMASZEWICZ et al., 2017), e a análise de isótopos estáveis pode ser usada também para compreender origens geográficas e diferenças entre 
populações (RUBENSTEIN et al., 2004, MARTINELLI, 2009). O trabalho realizado por Lara et al. (2012) com as espécies $P$. unifilis e Podocnemis expansa na região do baixo Araguaia, indica através da análise de isótopos de nitrogênio posições tróficas diferentes, em relação a razão isotópica de carbono os resultados expressam que para ambas as espécies a fonte de energia basal é proveniente de espécies de plantas $C_{3}$.

As plantas tipo $C_{3}$ e $C_{4}$ diferem na eficiência com que cada uma consome nitrogênio e água, as $C_{4}$ evoluíram em ambientes com temperaturas mais elevadas, abertos e em sua maioria sendo ambientes áridos, caracterizadas como plantas que evoluíram de um ancestral $C_{3}$ (WANG et al., 2014). As plantas $C_{3}$ recebem essa denominação devida a sua via fotossintética, possuindo como primeiro produto estável um ácido orgânico com três carbonos (BELTRÃO et al., 2008). Os valores isotópicos de $\delta^{13} \mathrm{C}$ podem várias nas plantas $C_{3}$ de $-24 \%$ a - $38 \%$, já as plantas tipo $C_{4}$, são observados valores entre -11\%o e -15\%o (MARTINELLI et al., 2009).

Este estudo teve como objetivo demonstrar a utilidade da análise isotópica como auxilio ao esclarecimento de questões ecológicas. Para a espécie $P$. unifilis, tivemos a finalidade de indicar padrões de consumo, interações tróficas e determinadas funções que a espécie exerce no ecossistema e também determinar a amplitude do nicho isotópico, considerando diferentes áreas e sexo; verificar se existe sobreposição de nicho isotópico em relação às áreas estudadas; verificando se a espécie estudada nas diferentes áreas coletadas faz parte de um mesmo nível trófico.

\section{MATERIAIS E MÉTODOS}

A área de estudo compreende uma extensão aproximada de $240 \mathrm{~km}$ ao longo do rio Xingu e na foz do rio Bacajá, situados entre os municípios de Altamira e Senador José Porfirio (entre 3ำ40'13.6"S,

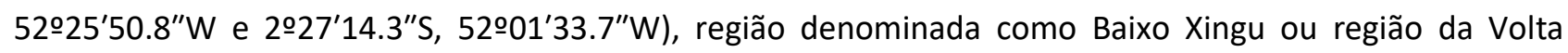
Grande, no estado do Pará, Brasil. Foram selecionadas quatro áreas de coletas previstas no Plano Básico Ambiental da UHE Belo Monte, essas áreas foram identificadas como A2, A3, A4 e A6 (Figura -1), os dados analisados foram cedidos pela empresa Norte Energia S.A, no âmbito da Licença de Operação no 1317/2015 da UHE Belo Monte.

O rio Xingu é um rio de águas claras e com pouco sedimento, em sua maioria é composto por muitas rochas e areia, fazendo com que suas águas sejam límpidas e transparentes em sua maior parte (Ayres, 1995). As suas nascentes estão localizadas em uma região de transição de biomas no estado de Mato Grosso, onde percorre parte do estado do Pará desaguando rio Amazonas na cidade de Porto de Moz (MOLINA, 2009; VELASQUEZ et al., 2010).

Três das áreas estudadas estão localizadas no rio Xingu, com exceção da área A6, localizada no rio Bacajá. As áreas do Xingu possuem fitofisionomia semelhantes, com florestas densas aluviais, porém atualmente são áreas que veem sofrendo com as interferências antrópicas, essas interferências alteram algumas características do rio e em alguns pontos a fitofisionomia marginal. A fitofisionomia do baixo Xingu é caracterizada por floresta ombrófila densa, floresta ombrófila aluvial, floresta ombrófila aberta com palmeira e floresta ombrófila aberta com cipós e palmeiras, florestas estacionais e savana (SALOMÃO, 2007). 
A área de estudo A2 é a mais a montante do rio Xingu, região com grande biodiversidade e áreas indígenas, a A3 é o local reconhecido regionalmente como Volta Grande do Xingu, as duas áreas possuem características semelhantes, tanto fitofisionômicas, em pluviosidade, turbidez, interferências antrópicas e também parâmetros físico-químicos (MIRANDA et al., 1988). A área A4 é a mais a jusante nas proximidades da foz do rio Xingu com o rio Amazonas, região de grandes profundidades, fauna diferenciada, grande diversidade de flora e altos riscos de predação de $P$. unifilis, nessa área fica localizado o tabuleiro do Embaubal uma das maiores e mais importantes áreas de reprodução para as espécies do gênero Podocnemis encontrados nessa região (CARNEIRO, 2012).

A área A6 difere em sua localização, situada no rio Bacajá, em aproximadamente $15 \mathrm{~km}$ da sua foz sentido a montante. O rio Bacajá é considerado um rio de águas brancas, isso devido à quantidade significativa de material (argila) em suspensão na coluna d'água, assim aumentando a turbidez e a quantidade de nutrientes.

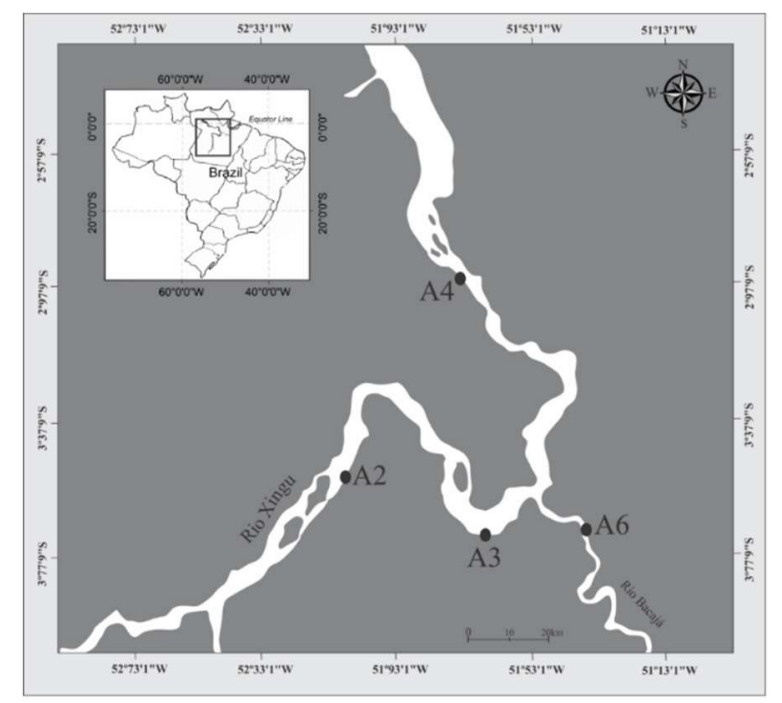

Figura 1: Mapa da região da Volta grande do Xingu.

Os indivíduos foram coletados no período de enchente (janeiro - março) do ano de 2017. O manejo e a captura dos quelônios seguiram o modelo previsto pelo manual de Manejo Conservacionista e Monitoramento Populacional de Quelônios Amazônicos (BALESTRA et al., 2016), foram usados os métodos de Redes de espera, Rede de arrasto, Mergulho e Coleta manual por avistamento ocasional. Foram coletadas amostras de folhas de vegetais que habitualmente são utilizadas como alimentação para a espécie $P$. unifilis. A identificação dos vegetais foi realizada por meio de entrevistas junto aos moradores das áreas estudadas, com foco nos pescadores, informando qual o tipo de vegetal é utilizado para isca e qual vegetal já foi avistado ocasionalmente sendo consumido pela $P$. unifilis. Sendo assim, foram coletadas amostras de nove tipos de plantas das famílias Araceae, Arecaceae, Asteraceae, Capparaceae, Fabaceae, Nymphaeaceae e Pontederiaceae (Tabela 2).

Para a análise isotópica foram retiradas amostras de tecidos (fragmento de epiderme) da membrana natatória das patas traseiras, posteriormente foram pesadas $(0,5$ a 0,6$)$ e as composições isotópicas $\left(\delta^{13} \mathrm{C}\right.$ e $\delta^{15} \mathrm{~N}$ ) determinadas a partir da combustão 'on-line' com a utilização de um Delta $\mathrm{V}^{\mathrm{tm}}$ Isotope Ratio Mass 
Spectrometer (IRMS) e um Organic Elemental Analyzer (OEA-Flash 2000) acoplado ao espectrômetro de massas no LARA - Laboratório de Radioecologia e Alterações Ambientais - do Instituto de Física (IF) da Universidade Federal Fluminense (UFF). O padrão utilizado para Carbono foi fóssil de Pee Dee Belemnite (PDB) e para o Nitrogênio o $N_{2}$ atmosférico (Lajtha \& Marshall, 1994) e expressos com relação ao padrão internacional por meio da equação: $\delta^{13} \mathrm{C}$ ou $\delta^{15} \mathrm{~N}=\left(\boldsymbol{R}_{\text {amostra }}-\boldsymbol{R}_{\text {padrão }} / \boldsymbol{R}_{\text {padrão }}\right) * 1000$, onde o R é razão correspondente de isótopos raros e comuns $\left({ }^{13} \mathrm{C} /{ }^{12} \mathrm{C} \mathrm{e}{ }^{15} \mathrm{~N} /{ }^{14} \mathrm{~N}\right)$.

Em geral, os valores isotópicos de $\delta^{13} \mathrm{C}$ e $\delta^{15} \mathrm{~N}$ detectados nos animais refletem os valores isotópicos de suas dietas (WADA et al., 1991; BEZERRA et al., 2015). Os isótopos de carbono são mais apropriados como marcadores, uma vez que fracionam muito pouco nas cadeias alimentares, ao contrário dos isótopos de nitrogênio que, pelas suas características, são mais utilizados em processos ecológicos (TIUNOV, 2007). O fracionamento isotópico pode ser caracterizado como enriquecimento ou empobrecimento da amostra em relação a sua fonte. Como exemplo, podemos utilizar estudos de sistemas fotossintéticos de plantas $C_{3}$, onde tem uma média de $-27,8 \%$ oxpondo ser $20 \%$ o mais negativa que o $\mathrm{CO}_{2}$ atmosférico $\left(\delta 13 \mathrm{C}_{\mathrm{CO} 2}=-7,4 \%\right.$ ), assim é possível entender como a matéria passa de um nível trófico a outro no ecossistema (PEREIRA, 2007).

Os dados analisados foram verificados primeiramente quanto a sua homogeneidade de variâncias através do teste Levene, indicando que as suposições foram atendidas. Posteriormente foi aplicado o teste ANOVA para verificar se existe a diferença entre as áreas e entre os sexos, referente à absorção de $\delta^{13} \mathrm{C} e$ $\delta^{15} \mathrm{~N}$, aplicando o teste Tukey para a confirmação das comparações entre as médias testadas.

\section{RESULTADOS}

Dos 63 indivíduos de $P$. unifilis coletados, foram coletadas medidas referentes ao tamanho dos indivíduos e peso, dentre a que melhor representa a diferença de tamanho dos espécimes é o comprimento da carapaça (CC). Os resultados obtidos pela diferenciação da morfologia entre 34 machos e 29 fêmeas foram apresentados conforme o teste estatístico ANOVA (Tabela 1).

Tabela 1: Dados descritivos (média, desvio padrão - SD, valor mínimo e máximo) do comprimento de carapaça (mm) e massa corpórea (g) de Podocnemis unifilis entre as áreas estudadas: A2 - Montante do Rio Xingu; A3 - Volta Grande; A4 - Jusante do Rio Xingu; A6 - Rio Bacajá.

\begin{tabular}{|c|c|c|c|c|c|c|c|c|}
\hline Áreas & $\mathbf{N}$ & MACHOS & & & $\mathbf{N}$ & FÊMEAS & & \\
\hline & & $\begin{array}{l}\begin{array}{l}\text { Média } \\
(\mathrm{mm})\end{array} \\
\end{array}$ & $\begin{array}{l}\text { Min } \\
(\mathrm{mm})\end{array}$ & PESO(g) & & $\begin{array}{l}\text { Média } \quad \pm \quad \text { DP } \\
(\mathrm{mm})\end{array}$ & $\begin{array}{l}\text { Min } \\
(\mathrm{mm})\end{array}$ & PESO (g) \\
\hline$A 2$ & 9 & $208.7 \pm 27.2$ & $179.0-250.0$ & $\begin{array}{l}864.0 \\
264.5 \\
\end{array}$ & 2 & $299.5 \pm 27.6$ & $280.0-319.0$ & $2380.0 \pm 622.3$ \\
\hline A3 & $\begin{array}{l}1 \\
4 \\
\end{array}$ & $218.4 \pm 55.0$ & $110.0-290.0$ & $\begin{array}{l}989.0 \\
595.0\end{array}$ & $\begin{array}{l}1 \\
3 \\
\end{array}$ & $283.5 \pm 79.0$ & $154.0-390.0$ & $\begin{array}{l}2331.0 \\
1410.0\end{array}$ \\
\hline A4 & 6 & $183.0 \pm 30.4$ & $152.0-241.0$ & $\begin{array}{l}595.0 \\
329.0\end{array}$ & 4 & $309.3 \pm 77.0$ & $215.0-390.0$ & $\begin{array}{l}3178.0 \\
1856.2\end{array}$ \\
\hline A6 & 5 & $215.0 \pm 38.0$ & $166.0-267.0$ & $\begin{array}{l}912.0 \\
469.3\end{array}$ & $\begin{array}{l}1 \\
0\end{array}$ & $298.2 \pm 48.0$ & $213.0-361.0$ & $2791.0 \pm 988.9$ \\
\hline
\end{tabular}

As médias de $\mathrm{CC}(\mathrm{mm})$ e do peso corporal não apresentaram variações significativas entre os machos, identificando maiores indivíduos (média $218.4 \pm 55.0 \mathrm{~mm}$ ) com maiores pesos corporais (média $989.0 \pm 595.0$ $\mathrm{mm}$ ) para a área $A 3$, já para a A4 apresentou o menor valor de CC (média $183.0 \pm 30.4 \mathrm{~mm}$ ), exibindo também 
os menores valores para a variável peso (média $595.0 \pm 329.0 \mathrm{~g}$ ).

As fêmeas obtiveram os maiores valores em CC (média $309.3 \pm 77.0 \mathrm{~mm}$ ) e também maiores proporções em peso (média $3178.0 \pm 1856.2 \mathrm{~g}$ ) na área A4. Os machos apresentaram um enriquecimento de $\delta^{13} \mathrm{C}$ e $\delta^{15} \mathrm{~N}$ proporcionais em todas as áreas, comparando o $\mathrm{CC}$ com a razão isotópica $\left(\delta^{13} \mathrm{C}-\mathrm{F}=6,69, \mathrm{P}=\right.$ 0,$001 ; \delta^{15} \mathrm{~N}-\mathrm{F}=0,987 \mathrm{P}=0,412$ ). Já as fêmeas obtiveram variações entre as áreas $\mathrm{A} 4$ e $\mathrm{A} 6$ para $\delta^{13} \mathrm{C}$, e variações de $\delta^{15} \mathrm{~N}$ entre as áreas $\mathrm{A} 2$ e $A 4,\left(\delta^{13} \mathrm{C}-\mathrm{F}=6,15, \mathrm{P}=0,002 ; \delta^{15} \mathrm{~N}-\mathrm{F}=3,61, \mathrm{P}=0,027\right)$ (Figura -2).

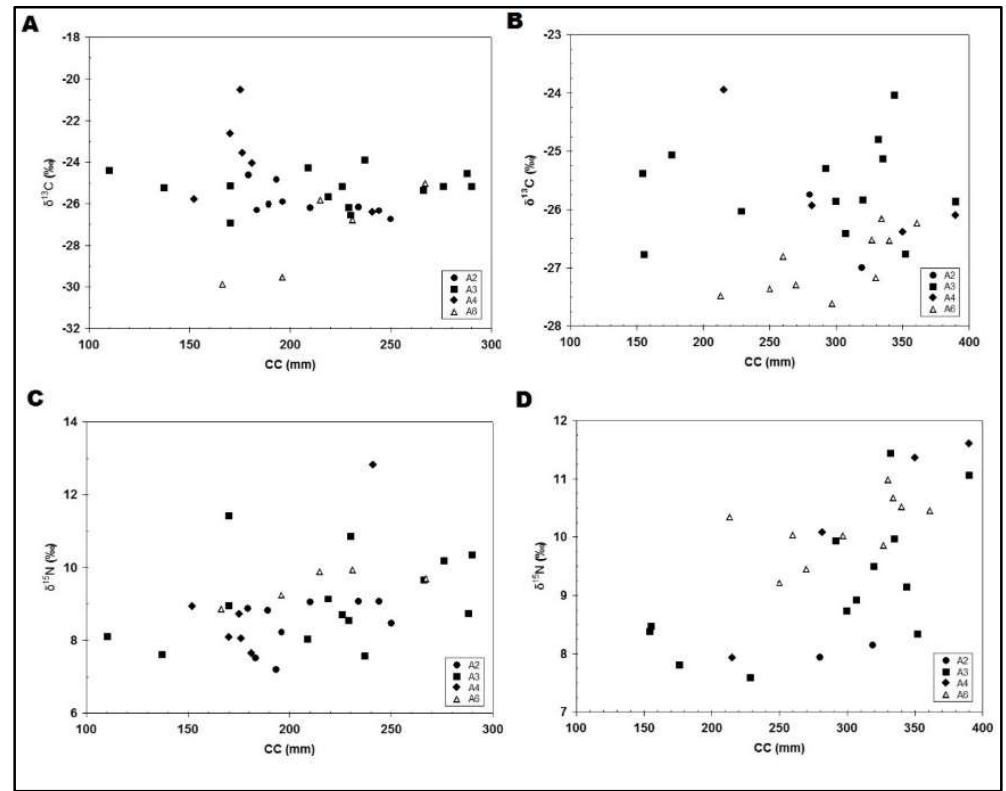

Figura 2: Índice de isótopos estáveis para Podocnemis unifilis em relação ao tamanho do corpo. Razões isotópicas (A machos $\delta 13 C$; B - fêmeas $\delta 13 C$; C - machos $\delta 15 N$; D - fêmeas $\delta 15 N$, todos em \%o) retiradas em amostras de pele.

Para os valores das razões isotópicas referentes aos grupos de indivíduos de cada área variaram quanto à média $( \pm S D)$, os valores médios referentes à $\delta^{13} \mathrm{C}( \pm S D)$ para as diferentes áreas não variaram tanto. Na A2 a razão se expressa em $-26.0 \%$ $\pm 0.7 \%$ (-27.0\%o a $-24.6 \%$ ), em A3 foram expressos em $-25.4 \%$ \pm 0.8\% (-26.9\% a -23.9\%o) representando resultados intermediários. A área A4 apresentou a maior média $24.5 \%$ $\pm 1.9 \%$ o (-26.4\% a $20.5 \%$ o), enquanto a $A 6$ apresenta o menor valor médio entre as áreas, sendo $27.1 \%$ $\pm 1.3 \%$ o $(-27.9 \%$ a $-25.0 \%$ o). Através da análise de variância foi possível identificar diferenças entres as áreas, onde os valores para a $\delta^{13} \mathrm{C}(\mathrm{F}=10,99, \mathrm{P}=0,000)$ e $\delta^{15} \mathrm{~N}(\mathrm{~F}=4,29, \mathrm{P}=0,008)$ comprovados através do teste da diferença totalmente significativa (TSD - Tukey Significant Difference).

De acordo com a análise isotópica realizada nas folhas dos vegetais coletados (Tabela 2), as nove espécies apresentaram valores de $\delta^{13} \mathrm{C}$ dentro dos valores citados na literatura para plantas $C_{3}(-32,08 \%$ o a $27,29 \%$ ) , fazendo parte da alimentação basal de $P$. unifilis, assim como a média dos valores dos vegetais para $\delta^{15} \mathrm{~N}(4,51 \% \circ \pm 3,47 \%)$.

Os valores referentes à $\delta^{15} \mathrm{~N}$ para a $\mathrm{A} 2$ são de $8.4 \%$ ๓ $0.7 \%$ (7.2\% to $9.1 \%$ ) apresentando menores valores em $\mathrm{A} 3$ de $9.2 \%$ o $\pm 1.2 \%$ \% (8.0\% to $11.4 \%$ ) , a A4 mostra valores médios $9.5 \%$ o $\pm 1.8 \%$ \% (7.6\% to $12.8 \%$ ), a A6 exprime à média ( \pm SD) de $10.0 \%$ \$ $1.0 \%$ ( $9.0 \%$ to $11.0 \%$ ) exibindo os maiores valores (Tabela 3). 
Tabela 2: Valores dos isótopos estáveis $\left(\delta^{13} \mathrm{C}, \delta^{15} \mathrm{~N}\right)$ para as amostras de plantas utilizadas na alimentação da $P$. unifilis na região do Baixo Xingu - PA.

\begin{tabular}{|l|l|l|l|l|l|l|}
\hline Família & Nome Científico & Nome comum & $\mathbf{\% C}$ & $\mathbf{\%} \mathbf{N}$ & $\boldsymbol{\delta}^{\mathbf{1 3}} \mathbf{C}(\% \mathbf{)}$ & $\boldsymbol{\delta}^{\mathbf{1 5}} \mathbf{N}(\% \mathbf{)}$ \\
\hline Fabaceae & Campsiandra laurifolia Benth. & Acapurana & 43,52 & 2,92 & $-29,07$ & 3,27 \\
\hline Araceae & Montrichardia arborescens (L.) Schott. & Aninga & 42,79 & 2,44 & $-29,05$ & 6,38 \\
\hline Fabaceae & Macrolobium acaciifolium (Benth.) Benth. & Arapari & 52,49 & 1,57 & $-29,96$ & 4,94 \\
\hline Fabaceae & Vatairea guianensis Aubl. & Fava-bolacha & 48,83 & 1,57 & $-30,97$ & 2,00 \\
\hline Capparaceae & Cratava tapia & Trapiá & 49,09 & 2,89 & $-29,54$ & 9,31 \\
\hline Asteraceae & Mikania sp. & Rama & 43,79 & 4,05 & $-31,01$ & 10,07 \\
\hline Arecaceae & Astrocaryum jauary Mart. & Jauarí & 48,36 & 1,18 & $-32,08$ & 1,92 \\
\hline Nymphaeaceae & Nymphaea gardneriana Planch. & Aguapé & 41,64 & 2,67 & $-27,29$ & $-1,51$ \\
\hline Pontederiaceae & Eichhornia crassipes (Mart.) Solms & Mururé & 48,26 & 1,45 & $-31,46$ & 4,23 \\
\hline
\end{tabular}

Tabela 3: Valores médios do isótopo estável $\left(\delta^{13} \mathrm{C}, \delta^{15} \mathrm{~N}\right)$ para amostras de pele de tracajá coletadas em 2017 nas áreas: A2 - Montante do Rio Xingu; A3 - Volta Grande; A4 - Jusante do Rio Xingu; A6 - Rio Bacajá nas proximidades da região do Baixo Xingu - PA.

\begin{tabular}{|c|c|c|c|c|c|c|c|c|c|}
\hline \multirow[t]{2}{*}{ Áreas } & \multirow[t]{2}{*}{$\mathbf{N}$} & \multicolumn{2}{|l|}{ \%C } & \multicolumn{2}{|l|}{$\% N$} & \multicolumn{2}{|l|}{$\delta^{13} \mathrm{C}(\%)$} & \multicolumn{2}{|l|}{$\delta^{15} \mathrm{~N}(\%)$} \\
\hline & & Média \pm DP & Min - Máx & Média \pm DP & Min - Máx & Média \pm DP & Min - Máx & Média $\pm \mathrm{DP}$ & Min - Máx \\
\hline A2 & 11 & $44.0 \pm 1.4$ & $\begin{array}{ll}42.6 & 47.3\end{array}$ & $14.3 \pm 0.5$ & $13.5 \quad 15.2$ & $-26.0 \pm 0.7$ & $-27.0-24.6$ & $8.4 \pm 0.7$ & 7.29 .1 \\
\hline A3 & 27 & $43.5 \pm 2.1$ & $\begin{array}{ll}36.3 & 46.9 \\
\end{array}$ & $14.1 \pm 0.7$ & $\begin{array}{ll}11.5 & 14.8\end{array}$ & $-25.4 \pm 0.8$ & $\begin{array}{ll}-26.9 & -23.9\end{array}$ & $9.2 \pm 1.2$ & $8.0 \quad 11.4$ \\
\hline A4 & 10 & $42.4 \pm 0.6$ & $\begin{array}{ll}41.4 & 43.2 \\
\end{array}$ & $13.6 \pm 0.2$ & $\begin{array}{ll}13.3 & 13.1 \\
\end{array}$ & $-24.5 \pm 1.9$ & $-26.4 \quad 20.5$ & $9.5 \pm 1.8$ & $\begin{array}{ll}7.6 & 12.8 \\
\end{array}$ \\
\hline A6 & 15 & $43.0 \pm 0.5$ & $42.3 \quad 44.0$ & $13.8 \pm 0.2$ & $\begin{array}{ll}13.5 & 14.1 \\
\end{array}$ & $-27.1 \pm 1.3$ & $\begin{array}{ll}-27.9 & -25.0\end{array}$ & $10.0 \pm 1.0$ & $\begin{array}{ll}9.0 & 11.0\end{array}$ \\
\hline
\end{tabular}

Os testes estatísticos apresentam diferenças significativas para $\delta^{13} \mathrm{C}$ entre as áreas A2 / A4, A3 / A6 e A4 / A6, e para a $\delta^{15} \mathrm{~N}$ os testes indicam apenas diferenças significativas entre as áreas $A 2$ / A6, onde é identificada pela não sobreposição dos polígonos de representação do nicho isotópico da espécie $P$. unifilis (Figura- 3).

\section{DISCUSSÃO}

As diferenças na morfometria intersexual se evidenciam, pois, os indivíduos dessa espécie têm como característica o dimorfismo sexual, onde as fêmeas são predominantemente maiores que os machos e possuem diferenças de tamanho na fenda da placa anal, distância pré-cloacal e também na espessura e comprimento da cauda (FACHÍN-TERÁN et al., 2004). As diferenças intrasexuais, referente às fêmeas eram esperadas na área $A 4$, se destacando com as maiores medidas morfometricas, devido ser uma área de grande amplitude de nicho ecológico, considerada uma importante área de desova dessa espécie, assim como uma importante área de alimentação, proporcionando uma região adequada para que a $P$. unifilis exerça o nicho realizado (CARNEIRO, 2012; LARA et al., 2012).

Existe uma pequena diferença de enriquecimento de $\mathrm{C}$ e $\mathrm{N}$ nas fêmeas, isso se dá pela diferença no $\mathrm{N}$ amostral ou pela diferenciação dos recursos disponíveis e utilizados pela espécie em cada área. Comparado a média dos grupos de indivíduos de cada área, é possível constatar que os vegetais apresentam posição de nível trófica diferentes dos quelônios, considerando a taxa de 3\%o de transferência de níveis (MANETTA et al., 2003; MARSHALL et al., 2007; MARTINELLI et al., 2009). Entre todas as áreas estudadas a A2 pode ser considerada a área com menor interferência antrópica, as águas são claras e com pouco material particulado disponível na coluna d'agua, vegetação marginal abundante, com poucas áreas para desova e águas turbulentas (MIRANDA et al., 1988).

A área A6 se localiza no rio Bacajá, rio que possui águas turvas e com uma grande disponibilidade de 
material particulado disponível na coluna d'agua. $O \delta^{15} \mathrm{~N}$ para a $A 6$ mostra que a absorção dos indivíduos daquela região é mais abundante em nitrogênio (Tabela 3), sugerindo que os indivíduos sejam mais generalistas com seu modo alimentar ou um possível produto de interferências antrópicas na região, como exploração mineral nas terras indígenas Trincheira - Bacajá, fazendo com que haja o aumento de material particulado na coluna d'água do rio Bacajá (MONTOVANELLI, 2016). Em Martinelli et al. (2010) foi realizado um estudo referente a concentração de nitrogênio em rios da zona tropical (Amazonas) e rios da zona temperada (São Paulo), onde é teorizado que o aumento da concentração de nitrogênio se dá pela interferência antrópica constante.

Em Lara et al (2012), diferenciaram a dimensão do nicho isotópico entre as espécies $P$. unifilis e $P$. expansa, com base nesse estudo observamos que os indivíduos das duas áreas em questão (A2; A6) exploram nichos diferentes, isso por que são ambientes com características fisionômicas e fitofisionômicas diferentes. Já as áreas A3 e A4 são trechos do rio Xingu com uma diferenciação tanto para a fitofisionomia quanto para as batimetrias, a A3 denominada Volta Grande do Xingu vem sofrendo altas interferências antrópicas, que podem interferir na ecologia da espécie em questão.

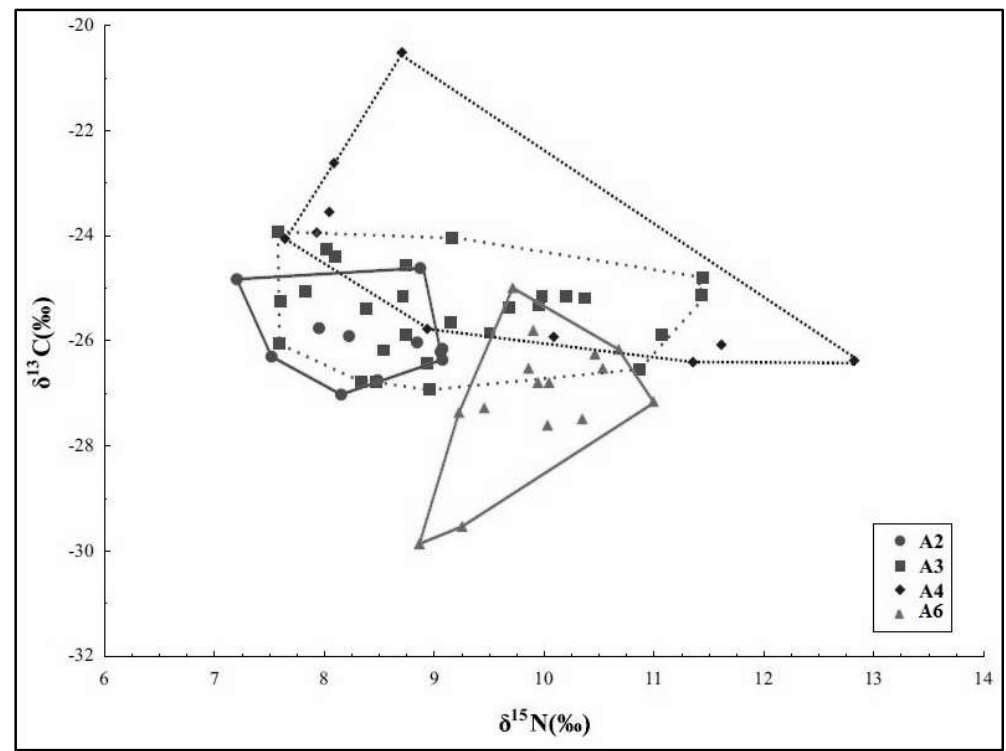

Figura 3: Nicho isotópico representado por polígonos em um $\delta$ espaço, formado pelas composições isotópicas dos indivíduos de P. unifilis das quatro áreas estudadas.

Estudos indicam que a transferência em nível trófico para $\delta^{15} \mathrm{~N}$ deve variar em média entre 2,5\%o (FRY, 1991) e 5\%o (ADAMS et al., 2000). Usando Lara et al. (2012) como referência, eles compararam as espécies $P$. expansa e $P$. unifilis encontrando uma diferença significativa de 2,5\%, indicando uma diferenciação da exploração de nicho isotópico e posição trófica das diferentes espécies. Neste estudo os valores encontrados para o $\delta^{15} \mathrm{~N}$ comumente utilizado para a identificação de transferência de nível trófico, não foram significativos para haver uma diferenciação (aproximadamente $1,6 \%$ ) entre os grupos das diferentes áreas estudadas. Porém essa diferenciação no fracionamento isotópico mostra uma característica diferente na ecologia dessa espécie, indicando uma capacidade adaptativa no seu desempenho de explorar e exercer o seu nicho ecológico, independente do ecossistema que ela se encontra. Essa capacidade adaptativa pode ser observada quanto o seu habito de desova, pois não demonstra ser uma espécie exigente, 
as fêmeas depositam seus ovos em ambientes mais rústicos, como pequenos bancos de areia, 'barrancos' e locais mais isolados com vegetação e diferentes tipos de substratos, usando como uma forma de defesa de sua ninhada ao contrário da P. expansa que apenas depositam seus ovos em grandes praias (MALVASío et al, 2005; NASCIMENTO, 2009).

\section{CONCLUSÕES}

Os isótopos estáveis funcionaram como uma importante ferramenta para auxiliar o entendimento da ecologia dos tracajás e o ecossistema onde habitam. Através do fracionamento isotópico foi possível identificar características da alimentação, nicho e adaptação dos indivíduos. Observamos neste estudo que existem variações nas razões isotópicas que podemos identificar como uma habilidade dessa espécie de se adaptar aos diferentes tipos de ambientes, sugerindo hábitos alimentares diversificados, proporcionais e adaptativos a cada área. Foi identificado através da $\delta^{13} \mathrm{C}$ que a base alimentar dessa espécie é composta por carbono fracionado de plantas $C_{3}$. Em relação à absorção de $\delta^{15} \mathrm{~N}$, não podemos afirmar que são populações diferentes ou que estão em níveis tróficos diferentes, pois as variações não foram suficientes em comparação a outros estudos. Para a exploração de outras áreas da ecologia dessa espécie, a fim de esclarecer questões maiores como especificidades de dietas e classificações em níveis tróficos comparados a outras espécies, será necessário fazer o uso de outros métodos complementares.

\section{REFERÊNCIAS}

ADAMS, T. S.; STERNER, R. W.. The effect of dietary nitrogen content on trophic level ${ }^{15} \mathrm{~N}$ enrichment. Limnol Oceanorg. The American Society of Limnology and Oceanography, v.45, n.3, p.601-607, 2000.

AYRES, J. M.. As matas de várzea do Mamirauá: médio Solimões. 2 ed. Brasília: CNPq, 1995.

BATES, H. W.. The Naturalist on the river Amazon. London: John Murray, 1892

BALESTRA, R. A. M.; VALADÃO, R. M.; VOGT, R. C.; BERNHARD, R.; FERRARA, C. R.; BRITO, E. S.; ARIAS, R. B.; MALVASIO, A.; LUSTOSA, A. P.; SOUZA, F. L.; DRUMMOND, G. M.; BASSETTI, L. A. B.; COUTINHO, M. E.; FERREIRA JUNIOR, P. D.; CAMPOS, Z. M. S.; MENDONÇA, S. H. S. T.; ROCHA, J. M. N.; LUZ, V. L. F.. Roteiro para inventários e monitoramentos de quelônios continentais. Biodiversidade Brasileira, v.6, n.1, p.114-152, 2016.

BEZERRA, M.; LACERDA, L.; REZENDE, C.; FRANCO, M.; ALMEIDA, M.; MACÊDO, G.; PIRE, T.; ROSTAN, G.; LOPEZ, G.. Food preferences and $\mathrm{Hg}$ distribution in Chelonia mydas assessed by stable isotopes. Environ. Pollut., v.206, p.236246, 2015.

BIASATTI, D. M.. Stable carbon isotopic profiles for sea turtle humeri: implications for ecology and physiology. Paleogeography, Paleoclimatology, and Paleoecology, v.206, p.203-216, 2004.

CARNEIRO, C. C.. Influência do ambiente de nidificação sobre a taxa de eclosão, a duração da incubação e a determinação sexual em Podocnemis (Reptilia, Podocnemididae) no Tabuleiro do Embaubal Rio Xingu, Pará. Dissertação (Mestrado em Ecologia Aquática e Pesca) Universidade Federal do Pará, Belém, 2012.

DENIRO, M. J.; EPSTEIN, S.. Influence of diet on the distribution of nitrogen isotopes in animals. Geochimica Cosmochimica Acta, v.45, p.341-331, 1981.

MATOS, J. V.. A pesca na Amazônia. Monografias Brasileiras III. Rio de Janeiro: Livraria Clássica de Alves, 1895.

FACHÍN-TERÁN, A.; VON MÜLHEN, E. M.. Reproducciónde la taricaya Podocnemis unifilis Troschel (1848) (Testudines: Podocnemididae) en la várzea del medio Solimões, Amazonas, Brasil. Ecología Aplicada, v.2, p.125-132, 2003.

FACHÍN-TERÁN, A.; VOGT, R. C.. Estrutura populacional, tamanho e razão sexual de Podocnemis unifilis (Testudines, Podocnemididae) no rio Guaporé (RO), norte do Brasil. Phyllomedusa: Journal of Herpetology, v.3, n.1, p.29, 2004.

HATASE, H.; TAKAI, N.; MATSUZAWA, Y.; SAKAMOTO, W.; OMUTA, K.; GOTO, K.; ARAI, N.; FUJIWARA, T.. Size-related differences in feeding habitat use of adult female loggerhead turtles Caretta caretta around Japan determined by stable isotope analyses and satellite telemetry. Marine Ecology Progress Series, v.233, p.273-281, 2002.

HOBSON, K. A.; CLARK, R. G.. Assessing avian diets using stable isotopes II: factors influencing diet-tissue fractionation. Condor 94, 1992. 
JUNK, W. J.; WANTZEN, K. M.. The flood pulse concept: new aspects, approaches and applications - an update. 2004.

MARSHALL, J. D.; RENÉE, B. J.; LAJTHA, K.. Sources of variation in the stable isotopic compositions of plants. In: LAJTHA, K.; MICHENER, R. H.. Stable isotopes in Ecology and Environmental Science. London: Blackwell Scientific Publications, 2007. p.22-50.

LARA, N. R. F.; MARQUES, T. S.; MONTELO, K. M.; ATAÍDES Á. G.; VERDADE, L. M.; MALVÁSIO, A.; CAMARGO, P. B.. A trophic stud of the sympatric Amazonian freshwater turtles Podocnemis unifilis and Podocnemis expansa ( Testudines, Podocnemidae ) using carbon and nitrogen stable isotopes analyses. NRC Research Press, 2012.

LEMONS, G.; LEWISON, R.; KOMOROSKE, L.; GAOS, A.; LAI, C.; DUTTON, P.; EGUCHI, T.; LEROUX, R.; SEMINOFF, J. A.. Trophic ecology of green sea turtles in a highly urbanized bay: Insights from stable isotopes and mixing models. Journal of Experimental Marine Biology and Ecology, v.405, n.1-2, p.25-32, 2011

MALVÁSIO, A.; SALERA JUNIOR, G.; SOUZA, A. M.; MODRO, N. R.. Análise da interferência do manuseio dos ovos no índice de eclosão e no padrão de escutelação do casco e as correlações encontradas entre as medidas das covas, ovos e filhotes em Podocnemis expansa (Schweigger, 1812) e Podocnemis unifilis (Troschel, 1848) (Testudines, Podocnemididae). Publicações Avulsas do Instituto Pau Brasil de História Natural, v.8, n.10, p.15-38, 2005.

MANETTA, G. L.; BENEDITO-CECILIO, E.. Aplicação da técnica de isótopos estáveis na estimativa da taxa de turnover em estudos ecológicos: uma síntese. Acta Scientiarum: Biological Sciences, Marigá, v.25, n.1, p.121-129, 2003.

MARTINELLI, L. A.; OMETTO, J. P. H. B.; FERRAZ, E. S.; VICTORIA, R. L.; CAMARGO, P. B.; MOREIRA, M. Z. Desvendando Questões Ambientais com Isótopos Estáveis. São Paulo: Oficina de Textos, 2009.

MARTINELLI, L. A.; COLETTA, L. D.; RAVAGNANI, E. C.; CAMARGO, P. B.; OMETTO, J. P. H. B.; FILOSO, S.; VICTORIA, R. L.. Dissolved nitrogen in rives: comparing pristine and impacted regions of Brazil. Brazilian Journal of Biology, v.70, n.3, p.709-722, 2010.

MIRANDA, E. E.; MIRANDA, J. R.; SANTOS, E. P. F.. Efeitos ecológicos das barragens do Xingu: uma avaliação preliminar. In: LEINAD, A.; SANTOS, O.; ANDRADE, L. M. M.. As hidrelétricas do xingu e os povos indígenas. São Paulo: Comissão Pró-Índio de S. Paulo, 1988.

MOLINA, J.. Questões hidrológicas no EIA Belo Monte. In: Painel de Especialistas: Análise Crítica do Estudo de Impacto Ambiental do Aproveitamento Hidrelétrico de Belo Monte, International Rivers. 2009. p.95-107.

NASCIMENTO, S. P.. Observações sobre o comportamento de nidificação de três espécies de Podocnemis Wagler (Testudinata, Pelomedusidae) no Baixo Rio Branco, Roraima, Brasil. Revista Brasileira de Zoologia, v.19, n.1, p.201-204, 2009.

PANTOJA-LIMA, J.; ARIDE, P. H. R.; OLIVEIRA, A. T.; FÉLIXSILVA, D.; PEZZUTI, J. C. B.; REBÊLO, G. H.. Chain of commercialization of Podocnemis spp. turtles (Testudines: Podocnemididae) in the Purus River, Amazon basin, Brazil: Current status and perspectives. J. Ethnobiol. Ethnomed, v.10, n.8, 2014

PEREIRA, A. L.. Isótopos estáveis em estudos ecológicos: métodos, aplicações e perspectivas. Revista Biociências, v.13, n.1-2, p.16-27, 2007

PORTELINHA, T. C. G.. Estrutura populacional e alometria reprodutiva de Podocnemis expansa (Testudines, Podocnemididae) no entorno do Parque Nacional do Araguaia, Tocantins. Dissertação (Mestrado em Ecologia Aplicada) - Universidade de São Paulo, Piracicaba, 2010.

RAMOS, R.; GONZÁLEZ-SOLÍS, J.; RUIZ, X.. Linking isotopic and migratory patterns in a pelagic seabird. Ecosystem Ecology, v.160, n.1, p.97-105, 2009.

REBÊLO, G.; PEZZUTI, J.. Percepções sobre o consumo de quelônios na Amazônia: sustentabilidade e alternativas ao manejo atual. Ambiente \& Sociedade, n.6-7, p.85104, 2000.

REBÊLO, G. H.; LUGLI, L.. The Conservation of Freshwater Turtles and the Dwellers of the Amazonian Jaú National Park (Brazil). In: JAIN, S. K.. Etnobiology in Human Welfare. Lucknow: Deep Publications, 1996.

RUBENSTEIN, D. R.; HOBSON, K. A.. From birds to butterflies: animal movement patterns and stable isotopes. Trends Ecol. Evol., v.19, n.256, 2004

SALOMÃO, R. P.; VIEIRA, I. C. G.; SUEMITSU, C.; ROSA, N. A.; ALMEIDA, S. S.; AMARAL, D. D.; MENEZES, M. P. M.. As florestas de Belo Monte na grande curva do rio Xingu, Amazônia Oriental. Ciências Naturais, n.3, p.57-153, 2007.

SAMPSON, A.; GIRALDO, A.; PAYAN, L. F.; AMOROCHO, D. F.; RAMOS, M. A.; SEMINOFF, J. A.. Trophic ecology of green turtle Chelonia mydas juveniles in the Colombian Pacific. Marine Biological Association of the United Kingdom. Journal of the Marine Biological Association of the United Kingdom, v.98, n.7, p.1817-1829, 2017.

SEMINOFF, J. A.; BJORNDAL, K. A.; BOLTEM, A. B.. Stable Carbon and Nitrogen Isotope Discrimination and Turnover in Pond Sliders Trachemys scripta: Insights for Trophic Study of Freshwater Turtles. Copeia. The American Society of Ichthyologists and Herpetologists, n.3, p.534-542, 2007.

TIUNOV, A. V.. Stable isotopes of carbon and nitrogen in soil ecological studies. Biology Bulletin, v.34, n.4, p.395-407, 2007.

TOMASZEWICZ, C. N. T.; SEMINOFF, J. A.; PRICE, M.; KURLE, C. M.. Stable isotope discrimination factors and betweentissue isotope comparisons for bone and skin from captive and wild green sea turtles (Chelonia mydas ). Rapid Communications in Mass Spectrometry, v.31, n.22, p.19031914, 2017

VAN DIJK, P. P.; IVERSON, J.; RHODIN, A.; SHAFFER,H.; BOUR, R.. Turtle Taxonomy Working Group. Turtles of the World. 7 Ed. AnnotatedChecklist of Taxonomy, Synonymy, Distribution with maps, and Conservation Status. Chelonian Research Monographs, n.5, v.7, p.329-479, 2014. 
VANZOLINI, P. E.. On clutch size and hatching success of the South American turtles Podocnemis expansa (Schweigger, 1812) and P. unifilis (Troschel, 1848) (Testudines, Podocnemididae). Anais da Academia Brasileira de Ciências, v.75, p.415-430, 2003

VELASQUEZ, C.; QUEIROZ, H.; BERNASCONI, P.. Fique por dentro: a Bacia do Rio Xingu em Mato. São Paulo: Instituto Socioambiental, 2010.

Vogt, R. C.. Temperature controlled sex determination as a tool for turtle conservation. Chelonian Conservation and Biology, v.1, p.159-162, 1994.

WADA, E.; MIZUTANI, H.; MINAGAWA, M.. The use of stable isotopes for food web analysis. Critical Reviews in Food Science and Nutrition, v.30, n.3, p.361-371, 1991.

WANG, L.; CZEDIK-EYSENBERG, A.; BRUTNEL, T. P.. Comparative analyses of $\mathrm{C} 4$ and $\mathrm{C} 3$ photosynthesis in developing leaves of maize and rice. Nature Biotechnology, v.32, n.11, p.1158-1164, 2014

WALLACE, B. P.; SEMINOFF, J. A.; KILHAM, S. S.; SPOTILA, J. R.; DUTTON, P. H.. Leatherback turtles as oceanographic indicators: stable isotope analyses reveal a trophic dichotomy between ocean basins. Marine Biology, v.149, p.953-960, 2006

A CBPC - Companhia Brasileira de Produção Científica (CNPJ: 11.221.422/0001-03) detém os direitos materiais desta publicação. Os direitos referem-se à publicação do trabalho em qualquer parte do mundo, incluindo os direitos às renovações, expansões e disseminações da contribuição, bem como outros direitos subsidiários. Todos os trabalhos publicados eletronicamente poderão posteriormente ser publicados em coletâneas impressas sob coordenação da Sustenere Publishing, da Companhia Brasileira de Produção Científica e seus parceiros autorizados. Os (as) autores (as) preservam os direitos autorais, mas não têm permissão para a publicação da contribuição em outro meio, impresso ou digital, em português ou em tradução. 